\title{
EFFECT OF VARIABLE VISCOSITY ON MHD INCLINED ARTERIAL BLOOD FLOW WITH CHEMICAL REACTION
}

\author{
B. TRIPATHI ${ }^{*}$ and B.K. SHARMA \\ Department of Mathematics, BITS Pilani (Pilani Campus), Pilani \\ Rajasthan-333031, INDIA \\ E-mail: bhavya.tripathi@pilani.bits-pilani.ac.in
}

\begin{abstract}
In this paper, we present the mathematical study of heat and mass transfer effects on an arterial blood flow under the influence of an applied magnetic field with chemical reaction. A case of mild stenosis is considered in a non-tapered artery which is inclined at an angle $\gamma$ from the axis. The variable viscosity of the blood is considered varying with the hematocrit ratio. Governing non-linear differential equations have been solved by using an analytical scheme, homotopy perturbation method to obtain the solution for the velocity, temperature and concentration profiles of the blood flow. For having an adequate insight to blood flow behavior through a stenosed artery, graphs have been plotted for wall shear stress, velocity, temperature and concentration profiles with varying values of the applied magnetic field, chemical reaction parameter and porosity parameter. The results show that in an inclined artery, the magnitude of the wall shear stress at stenosis throat increases as values of the applied magnetic field increase while it reduces as the values of both the chemical reaction and porosity parameters increase. Contour plots have been plotted to show the variations of the velocity profile of blood flow as the values of the height of the stenosis as well as the influence of the applied magnetic field increase.
\end{abstract}

Key words: magnetohydrodynamical fluid (MHD), homotopy perturbation method (HPM), variable viscosity, chemical reaction.

\section{Introduction}

Blood delivers all the important substances, nutrients and oxygen from one body cell to the other body cells through arteries and veins. Arteries play an important role in the transportation of highly oxygenated blood from the heart to the other body parts. Systematic circulation of the blood all over the body through arteries helps to stabilize the temperature and $\mathrm{pH}$ scale, by providing the nourishment to the body. Extra deposition of fat inside the artery, constricts the arterial wall and directly affects the work function of the artery. The accumulation of substances in an artery is known as stenosis which changes hemodynamic conditions and flow patterns that were existing in the artery, early to catheterization [1], [2], [3]. This larger scale of coronary artery blockage causes heart attacks in the human body[4]. We deal with this type of biological system related problems in "Biomechanics", which studies the functions and structures of the living body with the help of mechanics [5]. Since it is directly related to the health of a human body, nowadays it has gained serious attention from researchers, physiologists, and clinical persons to study the arterial blood flow. In this field, Ellahi et al. [6] in their paper mathematically explained a model of arterial blood flow with composite Stenosis. Further, in this work, Pralhad et al. [7] presented a model for arterial stenosis and computed the flow variables such as shear stress and resistance for the affected wall.

Erythrocytes are rich in hemoglobin which is an iron-containing biomolecule. So in the presence of a magnetic field, red blood cells allocated in arteries show characteristics of a diamagnetic fluid [8]. A field in which we study the effects of the applied magnetic field on biological fluids is known as biomagnetic fluid

\footnotetext{
${ }^{*}$ To whom correspondence should be addressed
} 
dynamics (BFD) and today it has numerous proposed applications in bioengineering and medical sciences. Magnetohydrodynamics (MHD) differs from BFD in the sense that it deals with electrically conducting fluids with the presence of an applied magnetic field [9]. In order to investigate the effects of the magnetic field on blood flow, Tzirtzilakis [10] proposed a mathematical model for Newtonian blood flow and proposed that under the strong magnetic field effect, the flow rate of the blood flow reduces up to $40 \%$. Further, in the same direction, Srivastava [11] analyzed the motion of the steady blood flow in an inclined porous artery under the influence of an applied magnetic field and concluded that as effects of the magnetic field increase the velocity profile of the blood flow under given conditions shows a reverse behavior.

The porosity of the medium is a major characteristic of the artery for analysing the effects of different physical parameters on the blood flow. Considering blood as an unsteady pulsatile laminar flow of an incompressible non-Newtonian fluid Akbarzadeh [12] numerically simulated the velocity profile of MHD blood flow which flows through a porous blood vessel and found that the increasing porosity of arterial media reduces the velocity of the blood flow. Further, in his mathematical model, Eldesoky [13] studied the motion of an unsteady MHD pulsatile flow of blood through a porous medium in a stenotic channel with slip at permeable walls and reported that in the porous medium the velocity of blood flow decreases as the effects of the magnetic field parameter and depth of the stenosis height increase. Khaled and Vafai [14] proposed a model for heat transfer for defining the role of porosity in biological tissues. However, none of these studies considered the effects of variable viscosity as viscosity was assumed to be constant.

In blood, the fraction of packed cell volume differs from point to point. So in a real physiological system, the functional dependence of the blood viscosity is not constant; it may vary either with the hematocrit ratio or may depend upon the temperature and the pressure [15] of the artery. In this regard, taking functional dependence of blood viscosity on hematocrit Layak et al. [16] analyzed the movement of the unsteady viscous blood flow in a vascular tube with an overlapping constriction by calculating the wall shear stress, pressure distribution and flow rate over the artery and proposed that as the value of the hematocrit parameter increases the viscosity of the blood flow also increases. Further, Sinha and Misra [17] investigated the effects of variable viscosity with variable hematocrit on an MHD flow of blood through a dually stenosed artery. Makinde and Onyejekwe [18] studied the effects of heat transfer on a model of MHD generalized Couette flow with temperature dependent viscosity and reported that increasing the viscosity exponent of the blood flow increases the viscous force and slows down the motion of the fluid.

Recently, the study of the chemical reactions on blood has become quite interesting because of the quantitative prediction of blood rate. In order to analyze the effects of the chemical reaction parameter on blood flowing artery, Mekheimer et al. [19] presented a paper which investigates the influence of the chemical reaction parameter on the blood flow through a tapered artery having overlapped stenosis. Akbar [20] studied the effects of chemical reaction on the hyperbolic tangent fluid model of the blood flow in a tapered artery with stenosis. By treating blood as a second-grade fluid, Misra and Adhikary [21] presented a model to study the effects of both heat and mass transfer on the oscillatory MHD flow of blood under the influence of chemical reaction.

However, the effects of both heat and mass transfer on MHD blood flow of having variable viscosity with chemical reaction have received little attention in the literature. Hence, the present article with the help of governing non-linear partial differential equations analyzes the combined effects of heat and mass transfer on a Newtonian, steady, incompressible fluid flow. The analytical technique homotopy perturbation method [22] has been used to obtain the solutions for the wall shear stress, velocity, temperature and concentration of the blood flow. Their respective graphs have been plotted for different values of the physical parameters of the problem.

\section{The mathematical model}

Let us consider a two-dimensional flow of blood through a porous stenosed artery of length $L$, inclined at an angle $\gamma$ from the vertical axis. The flow is assumed to be axially symmetric, incompressible, biomagnetic, Newtonian fluid. Under the fully developed boundary conditions, the flow is subject to a uniform magnetic field applied just perpendicular to the direction of the inclined artery as displayed in Fig.1. 
The viscosity of the blood is assumed to vary in a radial direction with a variable hematocrit of density $\rho$. The shape of the artery is assumed to be cylindrical in which $\bar{u} \bar{v}$ and $\bar{w}$ represent the velocity components in $\bar{r}, \bar{\theta}$ and $\bar{z}$ directions, respectively. Under these assumptions the governing equations of the blood flow are as follows

$$
\begin{aligned}
& \frac{\partial \bar{u}}{\partial \bar{r}}+\frac{\bar{u}}{\bar{r}}+\frac{\partial \bar{w}}{\partial \bar{z}}=0, \\
& \rho\left[\bar{u} \frac{\partial \bar{u}}{\partial \bar{r}}+\bar{w} \frac{\partial \bar{u}}{\partial \bar{z}}\right]=-\frac{\partial \bar{P}}{\partial \bar{r}}+\frac{\partial}{\partial \bar{r}}\left[2 \mu(\bar{r}) \frac{\partial \bar{u}}{\partial \bar{r}}\right]+2 \frac{\mu(\bar{r})}{\bar{r}}\left[\frac{\partial \bar{u}}{\partial \bar{r}}-\frac{\bar{u}}{\bar{r}}\right]+\frac{\partial}{\partial \bar{z}}\left[\mu(\bar{r})\left(\frac{\partial \bar{u}}{\partial \bar{z}}+\frac{\partial \bar{w}}{\partial \bar{r}}\right)\right], \\
& \rho\left[\bar{u} \frac{\partial \bar{w}}{\partial \bar{r}}+\bar{w} \frac{\partial \bar{w}}{\partial \bar{z}}\right]=-\frac{\partial \bar{P}}{\partial \bar{z}}+\frac{\partial}{\partial \bar{z}}\left[2 \mu(\bar{r}) \frac{\partial \bar{w}}{\partial \bar{z}}\right]+\frac{1}{\bar{r}} \frac{\partial}{\partial \bar{r}}\left[\bar{r} \mu(\bar{r})\left(\frac{\partial \bar{u}}{\partial \bar{z}}+\frac{\partial \bar{w}}{\partial \bar{r}}\right)\right]-\sigma_{1} \bar{\mu}_{m}{ }^{2} H_{0}{ }^{2} \bar{w}+ \\
& +\rho g \alpha\left(\bar{T}-\bar{T}_{0}\right) \cos \gamma+\rho g \alpha\left(\bar{C}-\bar{C}_{0}\right) \cos \gamma-\frac{\mu(\bar{r})}{k_{1}} \bar{w} \\
& \rho c_{p}\left[\bar{u} \frac{\partial \bar{T}}{\partial \bar{r}}+\bar{w} \frac{\partial \bar{T}}{\partial \bar{z}}\right]=\frac{k}{\bar{r}} \frac{\partial}{\partial \bar{r}}\left(\bar{r} \frac{\partial \bar{T}}{\partial \bar{r}}\right)+\mu(\bar{r})\left(\frac{\partial \bar{w}}{\partial \bar{r}}\right)^{2}, \\
& \left(\bar{u} \frac{\partial}{\partial \bar{r}}+\bar{w} \frac{\partial}{\partial \bar{z}}\right) \bar{C}=D\left(\frac{\partial^{2} \bar{C}}{\partial^{2} \bar{r}}+\frac{1}{r} \frac{\partial \bar{C}}{\partial \bar{r}}+\frac{\partial^{2} \bar{C}}{\partial^{2} \bar{z}}\right)+\frac{D K_{T}}{T_{m}}\left(\frac{\partial^{2} \bar{T}}{\partial^{2} \bar{r}}+\frac{1}{r} \frac{\partial \bar{T}}{\partial \bar{r}}+\frac{\partial^{2} \bar{T}}{\partial^{2} \bar{z}}\right)-L\left(\bar{C}-\bar{C}_{0}\right)
\end{aligned}
$$

where $L\left(\bar{C}-\bar{C}_{0}\right)$ is the factor of the chemical reaction parameter. The meaning of other symbols appearing in the equations have been listed in the nomenclature.

The variable viscosity $\mu(\bar{r})$ of the blood flow is defined as

$$
\mu(\bar{r})=\mu_{0}(1+\lambda h(\bar{r}))
$$

where

$$
\hbar(\bar{r})=H\left[1-\left(\frac{\bar{r}}{d_{0}}\right)^{m}\right]
$$

assume that $\lambda H=H_{r}, H$ denotes the maximum hematocrit at the center of the artery, $\lambda$ is a constant which has a numerical value $2.5, m$ determines the exact shape of the velocity profile and $H_{r}$ is the hematocrit parameter. 


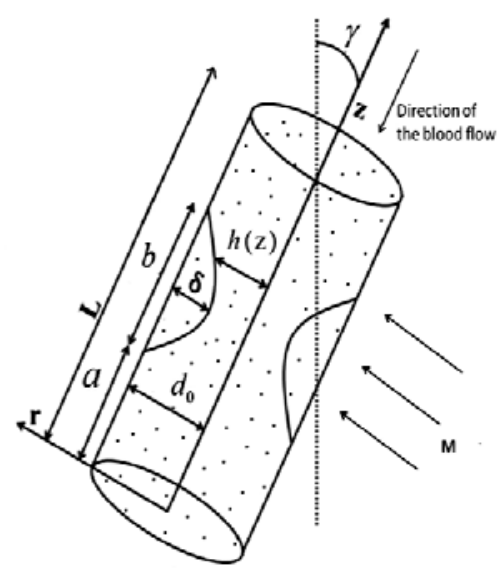

Fig.1. Geometry of the inclined artery with perpendicular applied magnetic field ( $M)$.

The geometry of the stenosis, located at a point $z$ with its maximum height of $\delta$, is defined by the formula [23]

$$
h(\bar{z})=\left\{\begin{array}{l}
d(\bar{z})\left[1-\eta\left(b^{n-1}(\bar{z}-a)-(\bar{z}-a)^{n}\right)\right] \text { when } a \leq \bar{z} \leq a+b \\
d(\bar{z}), \quad \text { otherwise, }
\end{array}\right.
$$

where $d(\bar{z})$ is the radius of the tapered artery in the stenotic region with

$$
d(\bar{z})=d_{0}+\xi \bar{z}
$$

in which $d_{0}$ represents the radius of the non-tapered artery and $\xi$ is the tapering parameter which defines by $\xi=\tan (\phi)$, where $\phi$ is known as the tapered angle. $\phi$ possess values just lower than zero $(\phi<0)$ for the case of converging tapered artery, for diverging tapered artery it takes the value greater than zero $(\phi>0)$ and the case of the nontapered artery $\phi$ has a zero value.

In Eq.(2.10), $\eta$ is defined by

$$
\eta=\frac{\delta^{*} n^{\frac{n}{n-1}}}{d_{0} b^{n}(n-1)}
$$

where $\delta^{*}$ is the maximum height of the stenosis located at

$$
\bar{z}=a+\frac{b}{n^{\frac{n}{n-1}}},
$$

and $n$ is the shape parameter which determines the shape of the constriction profile. Value $n=2$ results the symmetrically shaped stenosis and non-symmetric stenosis occurs for $n \geq 2$ values.

Now the nondimensional parameters are as follows 


$$
\left\{\begin{array}{l}
\bar{u}=\frac{u u_{0} \delta}{b}, \quad \bar{r}=r d_{0}, \quad \bar{z}=z b, \quad \bar{w}=w u_{0}, \quad \bar{h}=h d_{0}, \\
\bar{P}=\frac{u_{0} b \mu_{0} P}{d_{0}{ }^{2}}, \quad R_{e}=\frac{\rho b u_{0}}{\mu_{0}}, \quad \Theta=\frac{\left(\bar{T}-\bar{T}_{0}\right)}{\bar{T}_{0}}, \quad P_{r}=\frac{\mu c_{p}}{k}, \quad E_{c}=\frac{u_{0}{ }^{2}}{c_{p} T_{0}}, \\
Z=\frac{k_{1}}{d_{0}^{2}}, \quad M^{2}=\frac{\sigma H_{0}{ }^{2} d_{0}^{2}}{\mu_{0}} \quad S_{r}=\frac{\rho D k_{T} \bar{T}_{0}}{\mu_{0} T_{m} \bar{C}_{0}}, \quad S_{c}=\frac{\mu}{D_{p}}, \\
\sigma=\frac{\left(\bar{C}-\bar{C}_{0}\right)}{\bar{C}_{0}}, \quad E=\frac{\rho d_{0}{ }^{2} L}{\mu} .
\end{array}\right.
$$

The viscosity of the blood flow as given in Eq.(2.6) can be written in a non-dimensional form as

$$
\mu(r)=\mu_{0}\left(1+\lambda H\left(1-r^{m}\right)\right)
$$

In the case of mild stenosis $\left(\frac{\delta^{*}}{d_{0}} \ll 1\right)$ and by considering two other additional conditions [23]

$$
\frac{\operatorname{Re} \delta^{*} n^{\frac{1}{n-1}}}{b} \ll 1,
$$

and

$$
\frac{d_{0} n^{\frac{1}{n-1}}}{b} \sim O(1)
$$

Now, Eq.(2.1) to Eq.(2.5) change in a given nondimensional form, respectively, as

$$
\begin{aligned}
& \frac{\partial w}{\partial z}=0 \\
& \frac{\partial P}{\partial r}=0 \\
& \frac{\partial P}{\partial z}=\left[\frac{1}{r}+H_{r}\left(\frac{1}{r}-(m+1) r^{m-1}\right)\right] \frac{\partial w}{\partial r}+\left[1+H_{r}\left(1-r^{m}\right) \frac{\partial}{\partial r}\left(\frac{\partial w}{\partial r}\right)\right]+G_{r} \Theta \cos \gamma+ \\
& +G_{m} \sigma \cos \gamma-w\left[M^{2}+\frac{1}{Z}+\frac{H_{r}}{Z}\left(1-r^{m}\right)\right] \\
& \frac{1}{r} \frac{\partial}{\partial r}\left[r \frac{\partial \Theta}{\partial r}\right]+\mathrm{E}_{\mathrm{c}} \mathrm{P}_{\mathrm{r}}\left[\frac{\partial w}{\partial r}\right]^{2}=0,
\end{aligned}
$$




$$
\frac{1}{S_{c}}\left[\frac{1}{r} \frac{\partial}{\partial r}\left(r \frac{\partial \sigma}{\partial r}\right)\right]+S_{r}\left[\frac{1}{r} \frac{\partial}{\partial r}\left(r \frac{\partial \Theta}{\partial r}\right)\right]-E \sigma=0
$$

where $\mathrm{Br}=\mathrm{Ec} \operatorname{Pr}$ known as the Brinkman number which is the ratio of viscous heat generation to external heating and $E$ is the chemical reaction parameter.

The corresponding boundary conditions are as follows

$$
\begin{aligned}
& \frac{\partial w}{\partial r}=0, \quad \frac{\partial \Theta}{\partial r}=0, \quad \frac{\partial \sigma}{\partial r}=0 \quad \text { at } \quad r=0, \\
& w=0, \quad \Theta=0, \quad \sigma=0, \quad \text { at } \quad r=h(z)
\end{aligned}
$$

where $h(z)$ is the geometry of the stenosis in a non-dimensional form when the radius of the artery is of unit length $\left(d_{0}=1\right)$

$$
h(z)=\left\{\begin{array}{l}
\left(1+\xi^{\prime} z\right)\left[1-\eta_{1}\left(\left(z-l_{1}\right)-\left(z-l_{1}\right)^{n}\right)\right] \text { when } \quad l_{1} \leq z \leq l_{1}+1, \\
1, \quad \text { otherwise }
\end{array}\right.
$$

where

$$
\eta_{l}=\frac{\delta n^{\frac{n}{n-1}}}{(n-1)}, \quad \delta=\frac{\delta^{*}}{d_{0}}, \quad l_{1}=\frac{a}{b}, \quad \xi^{\prime}=\frac{\xi b}{d_{0}} .
$$

\section{Solution}

Now applying the homotopy perturbation method (HPM) to solve nonlinear differential Eqs (2.15)(2.19) under the given boundary conditions Eqs (2.20)-(2.21). In HPM, homotopies for velocity, temperature and concentration profiles are as follows

$$
\begin{aligned}
& H(q, w)=q\left[L(w)+H_{r}\left(\frac{1}{r}-(m+1) r^{m-1}\right) \frac{\partial w}{\partial r}+H_{r}\left(1-r^{m}\right) \frac{\partial}{\partial r}\left(\frac{\partial w}{\partial r}\right)-\frac{\partial p}{\partial z}\right]+ \\
& +(1-q)\left[L(w)-L\left(w_{0}\right)\right]-q\left[w\left(M^{2}+\frac{1}{Z}+H_{r} \frac{\left(1-r^{m}\right)}{Z}\right)-\cos \gamma\left(G_{r} \Theta+G_{m} \sigma\right)\right] \\
& H(q, \Theta)=(1-q)\left[L(\Theta)-L\left(\Theta_{0}\right)\right]+q\left[L(\Theta)+\operatorname{Ec} \operatorname{Pr}\left(\frac{\partial w}{\partial r}\right)^{2}\right] \\
& H(q, \sigma)=(1-q)\left[L(\sigma)-L\left(\sigma_{0}\right)\right]+q[L(\sigma)+\operatorname{Sc} \operatorname{Sr} L(\Theta)-\operatorname{Sc} E \sigma]
\end{aligned}
$$

where $L$ is the linear operator defined as

$$
L(X)=\frac{1}{r}\left(\frac{\partial}{\partial r}\left(r \frac{\partial X}{\partial r}\right)\right)
$$

The initial guesses which satisfy the corresponding boundary conditions (Eqs (2.20)-(2.21)) are given as 


$$
\begin{aligned}
& w_{10}=\frac{\left(r^{2}-h^{2}\right)}{4}\left(M^{2}+\frac{1}{Z}\right)\left(\frac{\partial p_{0}}{\partial z}\right), \\
& \Theta_{10}=\frac{\left(r^{2}-h^{2}\right)}{4}, \\
& \sigma_{10}=-\frac{\left(r^{2}-h^{2}\right)}{4} .
\end{aligned}
$$

Dependent variables can be decomposed in a series form as follows

$$
\begin{aligned}
& w(r, q)=w_{0}+q w_{1}+q^{2} w_{2}+O\left(q^{3}\right), \\
& \Theta(r, q)=\Theta_{0}+q \Theta_{1}+q^{2} \Theta_{2}+O\left(q^{3}\right), \\
& \sigma(r, q)=\sigma_{0}+q \sigma_{1}+q^{2} \sigma_{2}+O\left(q^{3}\right) .
\end{aligned}
$$

Substituting the series expansion form of $w(r, q), \Theta(r, q), \sigma(r, q)$ from Eqs (3.8)-(3.10) into Eqs (3.11)-(3.3) respectively, we compare the coefficients of $q^{0}, q^{1}$ and $q^{2}$.

For Eq.(3.12) coefficients of $q^{0}, q^{1}$ and $q^{2}$ are as follows

$$
\begin{aligned}
& q^{0}: \quad L\left(w_{0}\right)-L\left(w_{10}\right)=0 \Rightarrow w_{0}=w_{10}=\frac{\partial p_{0}}{\partial z} \frac{\left(r^{2}-h^{2}\right)}{4}\left(M^{2}+\frac{1}{Z}\right), \\
& q^{1}: \quad L\left(w_{1}\right)=-L\left(w_{0}\right)+\frac{\partial p_{0}}{\partial z}-H_{r}\left(\frac{1}{r}-(m+1) r^{(m-1)}\right) \frac{\partial w_{0}}{\partial r}-H_{r}\left(1-r^{m}\right) \frac{\partial}{\partial r}\left(\frac{\partial w_{0}}{\partial r}\right)+ \\
& +w_{0}\left(M^{2}+\frac{1}{Z}+H_{r} \frac{\left(1-r^{m}\right)}{Z}\right)-\cos \gamma\left(G_{r} \Theta_{0}+G_{m} \sigma_{0}\right), \\
& q^{2}: \quad L\left(w_{2}\right)=\frac{\partial p_{1}}{\partial z}-H_{r}\left(\frac{1}{r}-(m+1) r^{(m-1)}\right) \frac{\partial w_{1}}{\partial r}-H_{r}\left(1-r^{m}\right) \frac{\partial}{\partial r}\left(\frac{\partial w_{1}}{\partial r}\right)+ \\
& +w_{1}\left(M^{2}+\frac{1}{Z}+H_{r} \frac{\left(1-r^{m}\right)}{Z}\right)-\cos \gamma\left(G_{r} \Theta_{1}+G_{m} \sigma_{1}\right) .
\end{aligned}
$$

Similarly, for Eq.(3.2), coefficients of $q^{0}, q^{1}$ and $q^{2}$ are as follows

$$
\begin{aligned}
& q^{0}: \quad L\left(\Theta_{0}\right)-L\left(\Theta_{10}\right)=0 \Rightarrow \Theta_{0}=\Theta_{10}=\frac{\left(r^{2}-h^{2}\right)}{4}, \\
& q^{1}: \quad L\left(\Theta_{1}\right)=-L\left(\Theta_{0}\right)-\operatorname{Ec} \operatorname{Pr}\left(\frac{\partial w_{0}}{\partial r}\right)^{2},
\end{aligned}
$$




$$
q^{2}: \quad L\left(\Theta_{2}\right)=-2 \operatorname{Ec} \operatorname{Pr}\left(\frac{\partial w_{0}}{\partial r}\right)\left(\frac{\partial w_{1}}{\partial r}\right)
$$

For Eq.(3.3), coefficients of $q^{0}, q^{1}$ and $q^{2}$ are as follows

$$
\begin{array}{ll}
q^{0}: & L\left(\sigma_{0}\right)-L\left(\sigma_{10}\right)=0 \Rightarrow \sigma_{0}=\sigma_{10}=-\frac{\left(r^{2}-h^{2}\right)}{4}, \\
q^{1}: & L\left(\sigma_{1}\right)=-L\left(\sigma_{0}\right)-\operatorname{ScS} \operatorname{Sr} L\left(\Theta_{0}\right)+\operatorname{Sc} E \sigma_{0}, \\
q^{2}: & L\left(\sigma_{2}\right)=-\operatorname{ScSr} L\left(\Theta_{1}\right)+\operatorname{Sc} E \sigma_{1} .
\end{array}
$$

Now, with the help of the values $w_{0}, \Theta_{0}, \sigma_{0}$ from Eqs (3.5)-(3.7) and by using the definition the linear operator for $L\left(w_{0}\right), L\left(\Theta_{0}\right), L\left(\sigma_{0}\right)$ into Eqs (3.22), (3.15) and (3.18), we attain the expressions for $w_{1}, \Theta_{1}$ and $\sigma_{1}$ as follows

$$
\begin{aligned}
& w_{1}=\frac{\left(r^{2}-h^{2}\right)}{4}\left(\frac{\partial p_{0}}{\partial z}\right)\left(1-\left(M^{2}+\frac{1}{Z}\right)\right)-\frac{\cos \gamma}{64}\left(r^{4}+3 h^{4}-4 r^{2} h^{2}\right)\left(G_{r}-G_{m}\right)+ \\
& +\frac{H_{r}}{4 Z}\left(\frac{\partial p_{0}}{\partial z}\right)\left(M^{2}+\frac{1}{Z}\right)\left(\frac{r^{4}+3 h^{4}}{16}+\frac{\left(h^{m+4}-r^{m+4}\right)}{(m+4)^{2}}+\frac{h^{2} r^{m+2}-h^{m+4}}{(m+2)^{2}}\right)+ \\
& -\frac{H_{r}}{4 Z}\left(\frac{\partial p_{0}}{\partial z}\right)\left(M^{2}+\frac{1}{Z}\right)\left(\frac{r^{2} h^{2}}{4}\right)+\left(\frac{\partial p_{0}}{\partial z}\right)\left(M^{2}+\frac{1}{Z}\right)^{2}\left(\frac{r^{4}}{16}+\frac{3 h^{4}}{16}-\frac{h^{2} r^{2}}{4}\right)+, \\
& -\frac{H_{r}}{2}\left(\frac{r^{2}-h^{2}}{2}+\frac{h^{m+2}-r^{m+2}}{m+2}\right)\left(\frac{\partial p_{0}}{\partial z}\right)\left(M^{2}+\frac{1}{Z}\right), \\
& \Theta_{1}=-\frac{\left(r^{2}-h^{2}\right)}{4}-\operatorname{EcPr} \frac{\left(r^{4}-h^{4}\right)}{64} P^{2} U^{2}, \\
& \sigma_{1}=(1-\operatorname{ScSr}) \frac{\left(r^{2}-h^{2}\right)}{4}+\operatorname{Sc} E\left(\frac{r^{4}}{64}+\frac{3 h^{4}}{64}-\frac{r^{2} h^{2}}{16}\right) .
\end{aligned}
$$

After getting the expressions for, $\theta_{1}$ and $\sigma_{1}$ by following the same steps, we further calculate the values of $w_{2}, \Theta_{2}$ and $\sigma_{2}$ with the help of MATLAB-2015b. We get final expressions for velocity, temperature and concentration profiles of the blood flow by putting the values of all the calculated variables in Eqs (3.8)-(3.10, respectively.

\section{Results and discussions}

The motivation behind the research is to analyze the effects of heat and mass transfer on the blood flow through an inclined stenosed artery under the influence of the applied magnetic field with chemical reaction. A list of all the parameters used to graphically analyze the validity of the mathematical model is given in Tab.1, where the value of the height of the stenosis $(\delta)$ is considered 0.1 for the case of mild stenosis. 


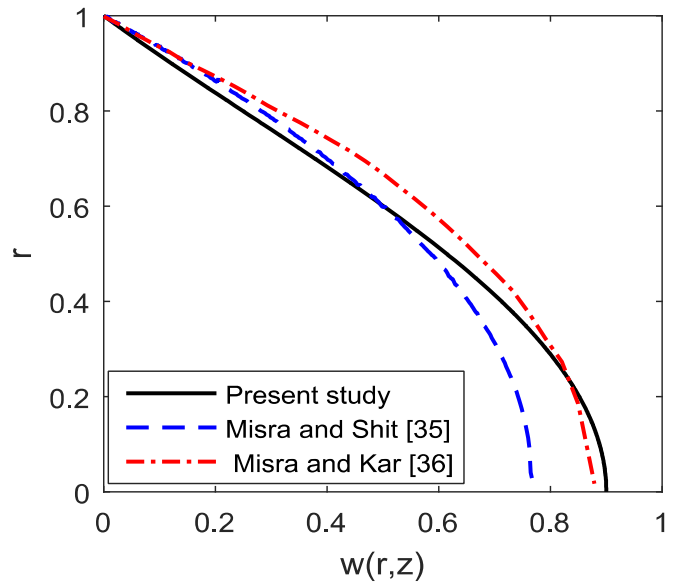

Fig.2. Comparision result of variation of velocity profile for a Newtonian model of blood flow

Table 1. Values of the parameters.

\begin{tabular}{|ccc|}
\hline Parameters & Values(Unit free) & Source \\
\hline The height of the stenosis $(\delta)$ & 0.1 & {$[11],[24]$} \\
The inclination angle of the artery $(\gamma)$ & $\frac{\pi}{6}$ & {$[25],[26]$} \\
Porosity parameter $(Z)$ & 0.3 & \\
Chemical reaction parameter $(E)$ & 1 & {$[27]$} \\
Shape parameter for symmetric case $(n)$ & 2 & {$[28]$} \\
Grashof Number $(\mathrm{Gr})$ & 2 & {$[29],[23]$} \\
Modified Grashof Number $\left(G_{m}\right)$ & 3 & {$[30]$} \\
Brinkaman number $(\mathrm{Br})$ & 2 & {$[30]$} \\
Hematocrit parameter $\left(H_{r}\right)$ & 1 & {$[31]$} \\
Magnetic field parameter $(M)$ & 1.5 & {$[32]$} \\
Schmidt number $(\mathrm{Sc})$ & 1 & {$[33],[34]$} \\
Soret number $(\mathrm{Sr})$ & 0.5 & {$[31]$} \\
Ratio of $\frac{a}{b}\left(l_{l}\right)$ & 0 & {$[31]$} \\
Location of the maximum height of the stenosis & 0.5 & {$[29]$} \\
\hline
\end{tabular}

Considering the case of mild stenosis Fig.2 provides result of a comparison between the present study and those reported in Misra and Shit [35] and in Misra and Kar [36] for the Newtonian fluid model of the blood flow. The present result shows a good agreement with those published by Misra and Shit and in Misra and Kar.

All the graphs are plotted for the range of 0 to 0.9 by assuming a non-stretching stenotic wall of the artery (as the value of the height of the stenosis for mild stenosis case is assumed as 0.1 ). All the figures have been plotted by using the parameters values as given in Tab.1. Figure 3 and Fig.4 display the radial variation of velocity and temperature profiles for different values of the inclination angle $(\gamma)$, made by the non-tapered artery from the vertical axis. The magnitude of the applied magnetic field is assumed to be same for each and every inclined position of the artery. It can be clearly observed from Fig. 3 that as the values of the inclination angle of the artery increase, the velocity profile of the blood flow decreases, respectively. It can be clearly seen from the figure that the pattern of all velocity profiles for different inclination angles are similar in the sense that they show the decrease in their maxima as one moves away from the center of the artery and finally fall to zero at the stenotic wall. From Fig.4 it is clear that as the value of the inclination angle of the artery increases from 0 to $\frac{\pi}{3}$, the temperature profile of the blood flow also increases. 


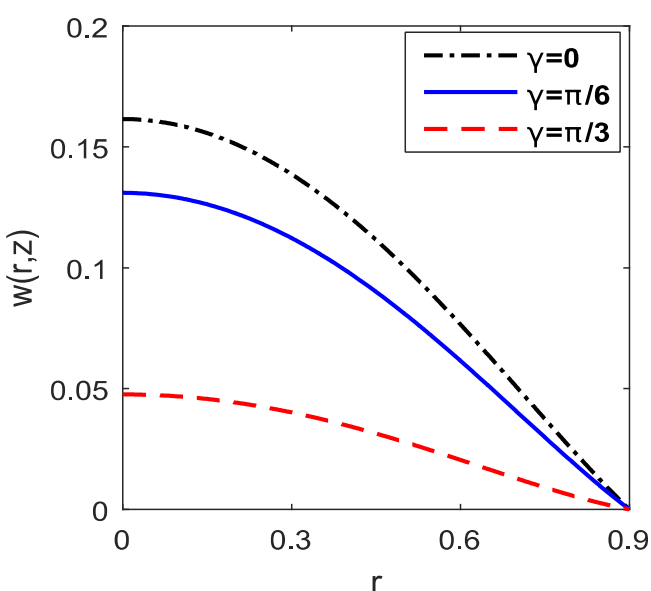

Fig.3: Radial distribution of velocity profile for different values of inclination angle $(\gamma)$.

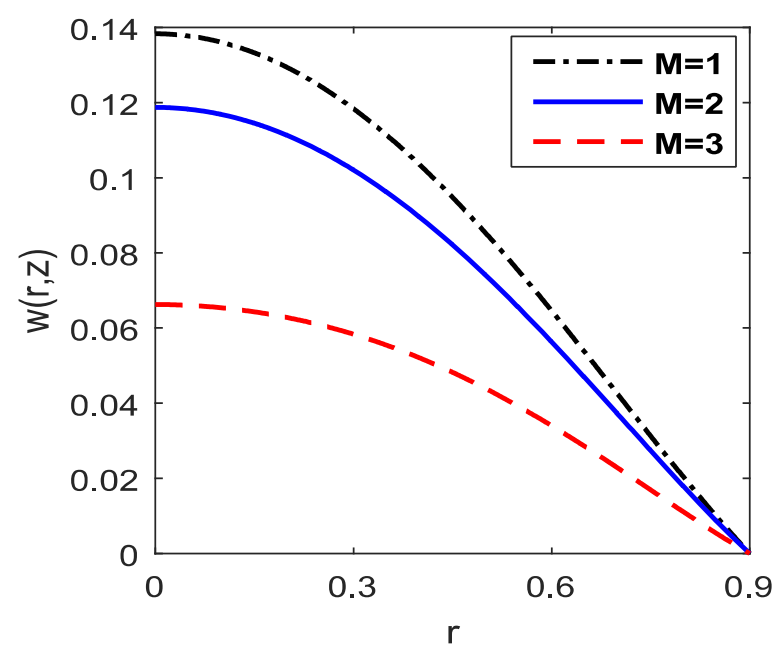

Fig.5. Radial distribution of velocity profile for different values of magnetic field parameter.

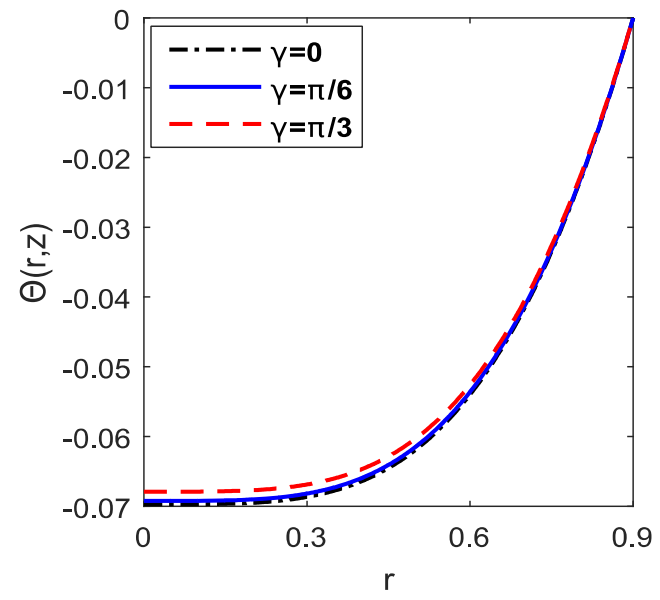

Fig.4. Radial distribution of temperature profile for different values of inclination angle $(\gamma)$.

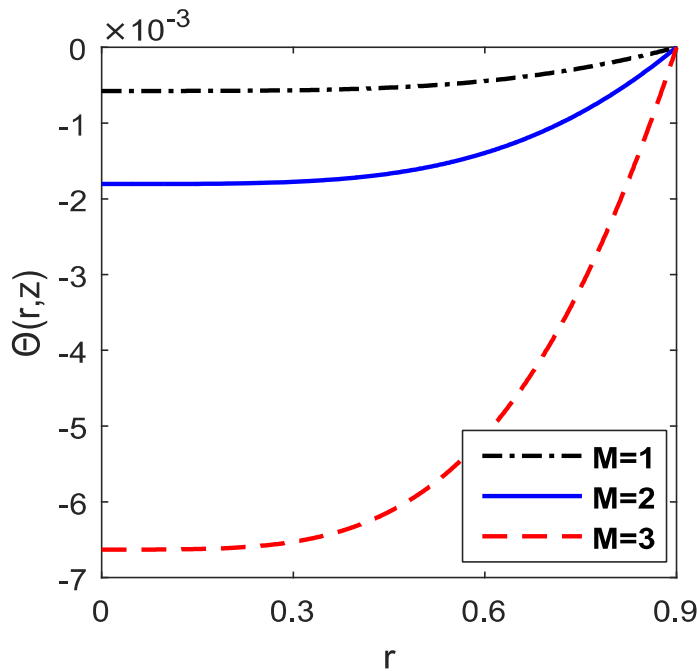

Fig.6. Variations of the temperature profile for different values of a magnetic field parameter.

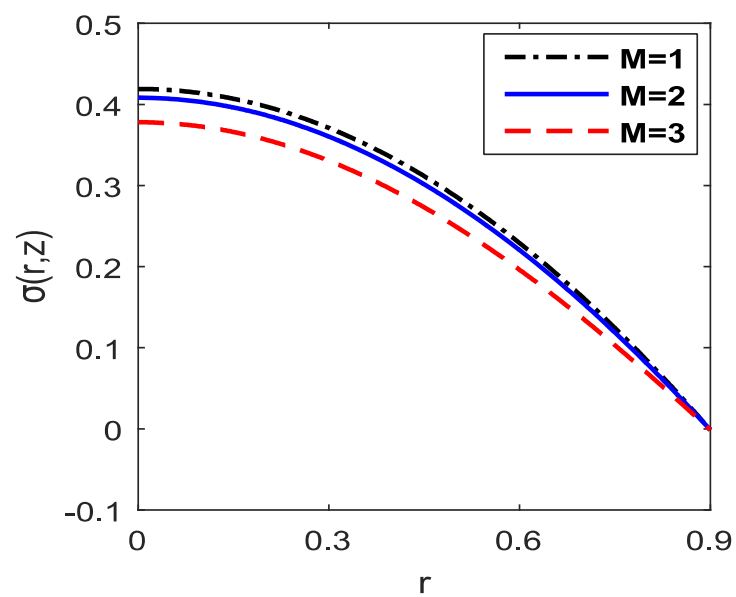

Fig.7. Radial distribution of concentration profile for different values of magnetic field parameter. 
Figure 5 illustrates the radial distribution of the velocity profile of the blood flow for different values of the magnetic field parameter $(M)$. One can notice the half-flattened parabolic velocity profile from the figure, which decreases as the values of the applied magnetic field increase. It happens because blood contains

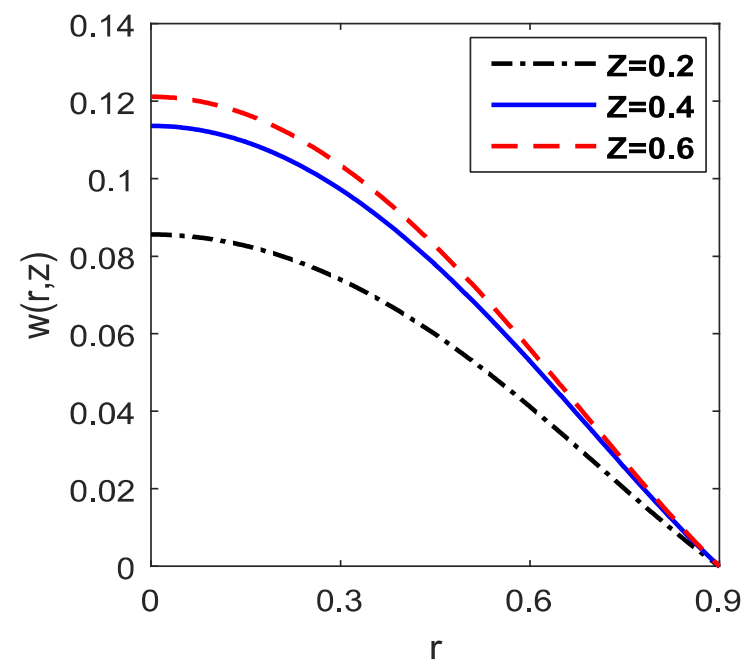

Fig. 8. Radial distribution of velocity profile different values of porosity parameter.

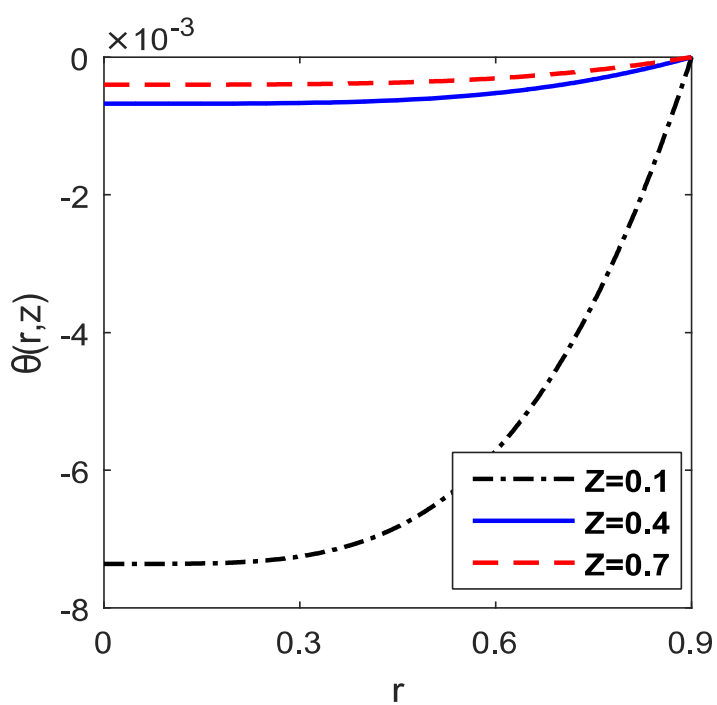

Fig.9. Radial distribution of temperature profile for different values of porosity parameter.

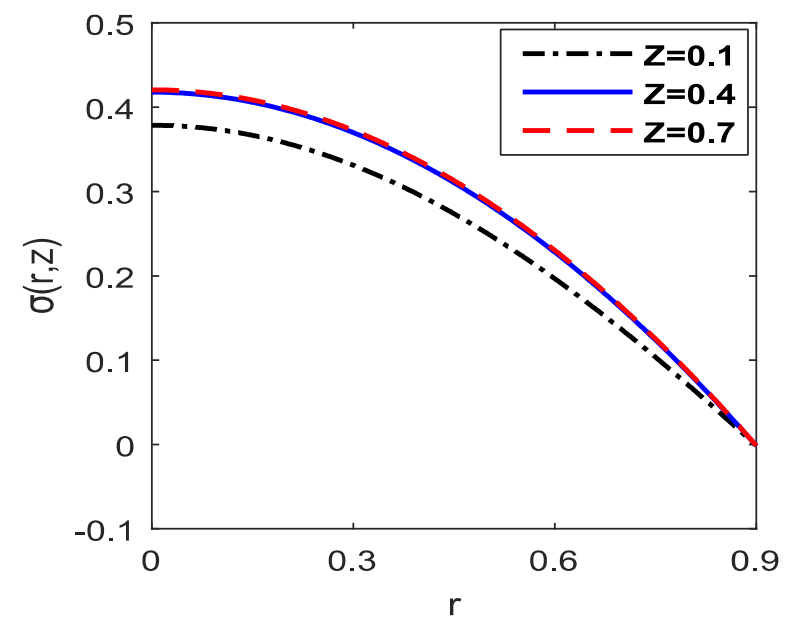

Fig.10. Radial distribution of concentration profile for different values of porosity parameter.

magnetic iron oxide particles and when blood flows under the action of the applied magnetic field, it feels a strong electromotive force. This effect of magnetization causes a rotational motion of charged particles and magnetic particles of the blood. This type of orientation in blood, form red blood cells and magnetic particles more suspended in the blood plasma and increase the value of blood viscosity and that directly affects the velocity of the blood flow. So as the value of the applied magnetic field parameter increases, Lorentz force which stabilizes between moving magnetic particles and the applied magnetic field opposes the motion of the blood flow and causes reduced velocity profile of the blood flow. The result for the velocity profile agrees well with the result reported by Sharma et al. [33]. The effect of the magnetic field parameter $(M)$ on 
temperature profile is displayed in Fig.6. It is noticed from the figure that temperature profile of the blood flow in an inclined artery decreases as the value of the applied magnetic field which works just perpendicular to the stenosed artery, increases. Figure 7 displays the effects on concentration profile of the blood flow as the intensity of the applied magnetic varies. From the figure it is clear that concentration profile of the blood flow under variable viscosity effect, decreases as the value of the applied magnetic field increases from 1 to 3 and for a particular value of the magnetic field parameter concentration of the blood in the inclined porous artery decreases from the center towards the arterial wall and falls to zero at stenosis wall.

Variations of velocity, temperature and concentration profiles of the blood flow for different values of the porosity parameter $(Z)$ have been analyzed with the help of Fig.8, Fig.9 and Fig.10, respectively. Figure 8 illustrates that as the values of the porosity parameter increase, the velocity profile of the blood flow also increases. It shows that for a particular value of the porosity parameter, velocity attains its maximum value at the middle of the artery and gradually starts decreasing towards the arterial wall. The velocity profile with the porosity parameter shows this behavior may be because, when a fraction of the voids volume over the total volume increases, it can be more possible for fluid particles to move from one place to another place in the artery. In the inclined artery, this can be the cause of decreased velocity with an increase in the porosity parameter. Similarly, Fig.9 and Fig.10 illustrate that the temperature and concentration profiles of the blood flow in the stenosed artery also increase as the value of porosity parameter increases.

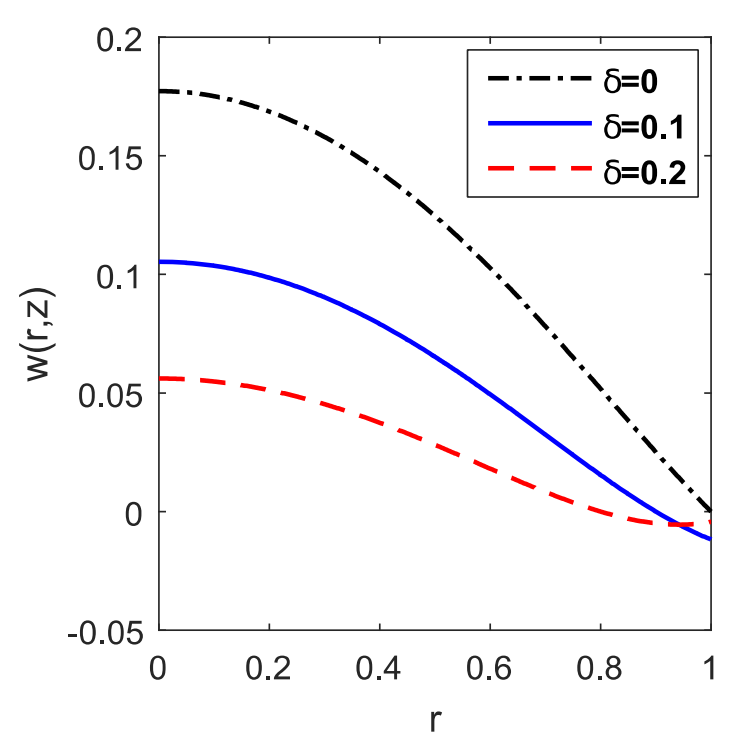

Fig.11. Radial variation of velocity profile for different values of stenotic height $(\delta)$.

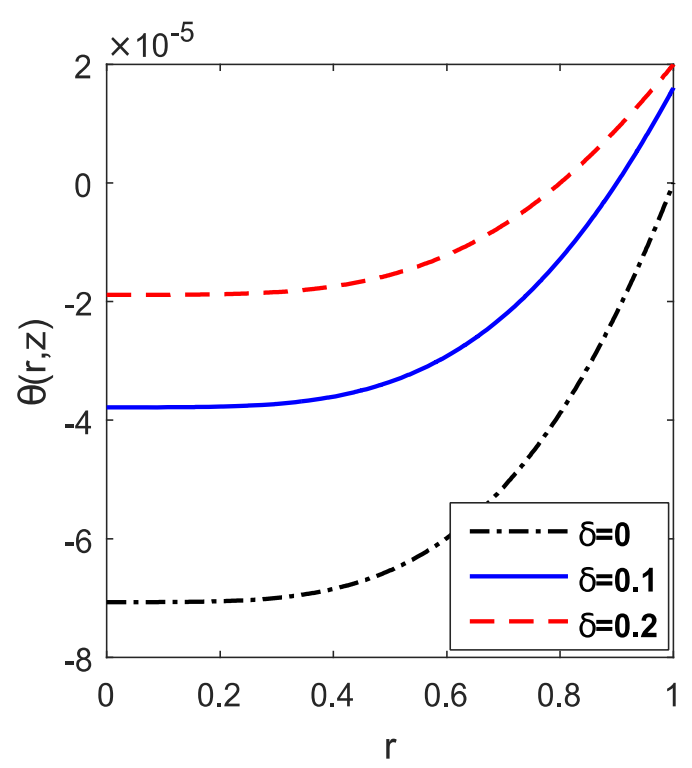

Fig.12. Radial variation of the temperature profile for different values of stenotic height $(\delta)$.

Figure 11 depicts the distribution of velocity profile of the blood flow with different sizes of the stenosis. It is clear from the figure that the velocity profile of the blood flow decreases as the stenosis in the artery increases in size. It may also be noted that the velocity profile decreases the onset of the stenosis towards the stenosis throat. This result is in good agreement with those reported by Misra and Shit [35]. Figure 12 displays the effects of the height of the stenosis $(\delta)$ on the variations of the temperature profile of the blood flow. It shows that the temperature profile of the blood flow increases as the value of the height of the stenosis increases from 0 to 0.2 . The first case where $\delta=0$ is considered to analyze the distribution of velocity and temperature profiles of the blood flow in the stenosed free artery. 


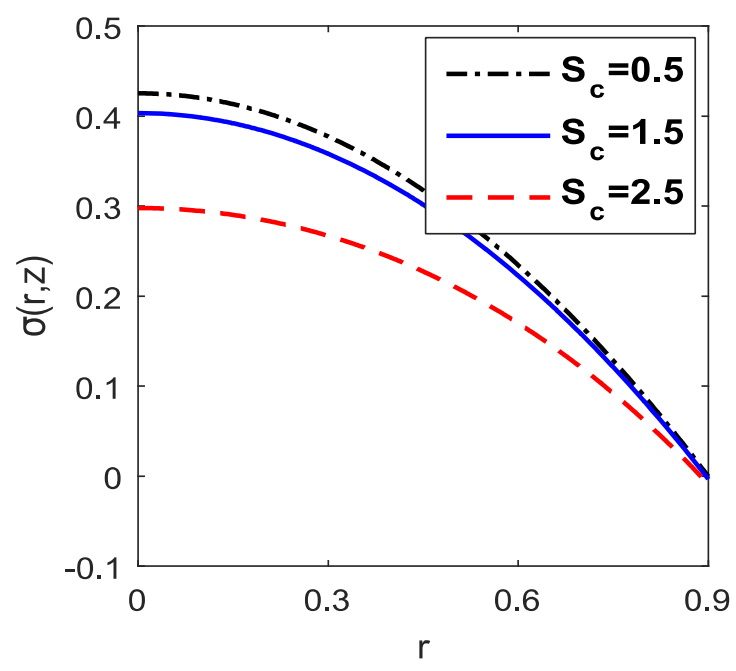

Fig.13. Radial variation of concentration profile for different values of Schmidt number $(\mathrm{Sc})$.

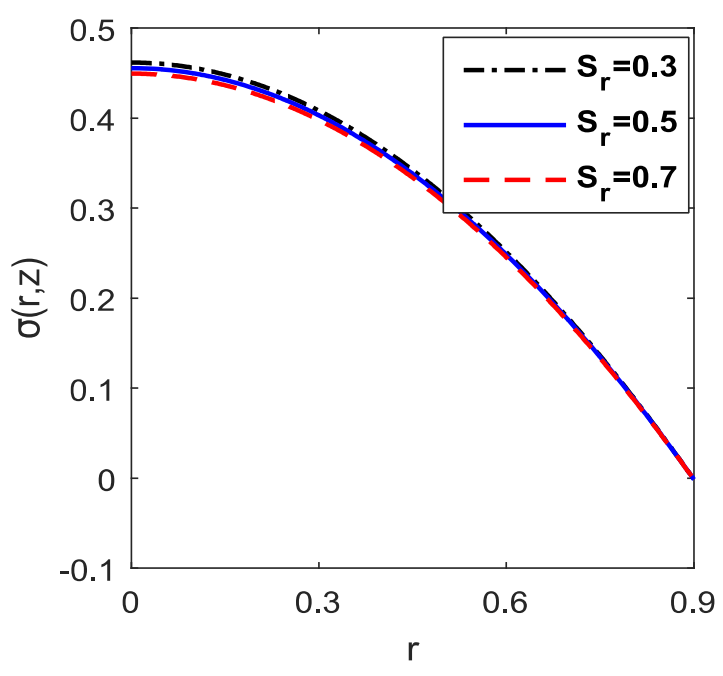

Fig.14. Radial variation of concentration profile for different values of Soret number (Sr).

Figure 13 and Fig.14 give the variation in concentration profiles of the blood flow with $r$ for different values of the Schmidt number (Sc) and the Soret number (Sr) respectively. Under the influence of chemical reaction, the concentration profile of the blood flow reduces as the effects of both the Schmidt and Soret number increase in the stenosed artery. A similar behavior of the concentration profile with changing values of the Schmidt number (Sc) has been observed by Kandasamy et al. [37].

\subsection{The expression for the shear stress} as follows

Shear stress in arteries is defined by the force per unit area on the arterial wall and it can be calculated

$$
\tilde{S}_{r z}=\left(\frac{\partial w}{\partial r}\right)
$$

expression for wall shear stress is

$$
\tilde{S}_{r z}=\left[\frac{\partial w}{\partial r}\right]_{r=h}
$$

Figure 15 displays the variations in shear stress profiles at stenosis throat for different values of the chemical reaction $(E)$ parameter. From the figure, it is clear that as the value of the chemical reaction parameter increases, shear stress at the stenosis throat of the artery reduces respectively. Further, for different values of the porosity and magnetic field parameters Figs 16 and 17 show the variations of the shear stress profile at the stenosis throat of an inclined porous artery. It can be clearly observed from these figures that as the values of the porosity parameter increase, the shear stress profile at the stenosis throat decreases and it also decreases with the increased value of the magnetic field parameter. 


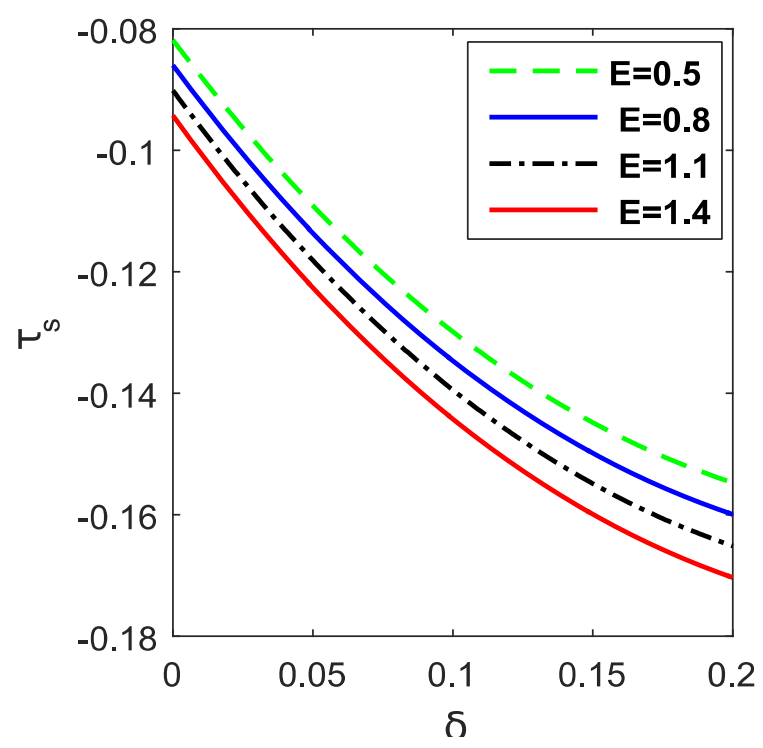

Fig.15. Variation of shear stress at the stenosis throat for different values of chemical reaction parameter $(E)$.

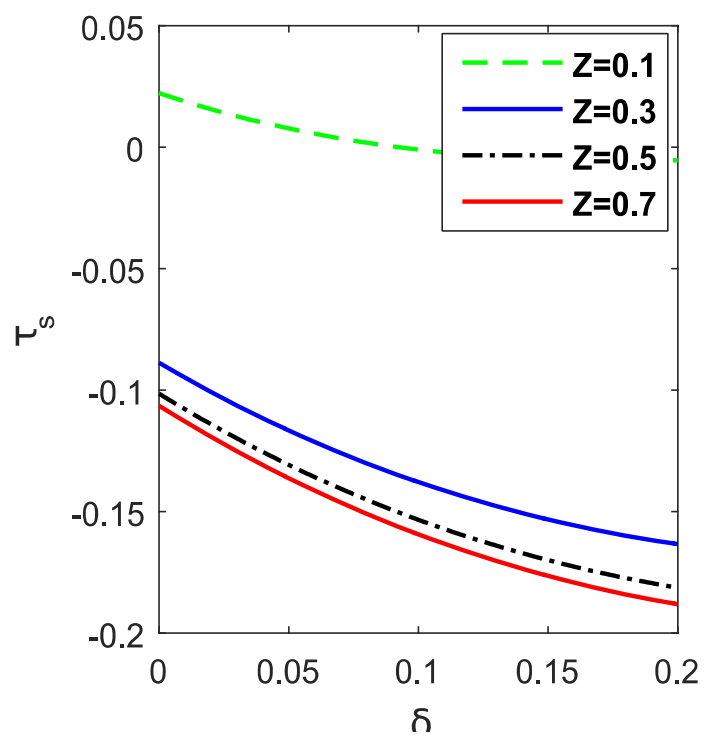

Fig.16. Variation of shear stress at the stenosis throat for different values of porosity parameter (Z).

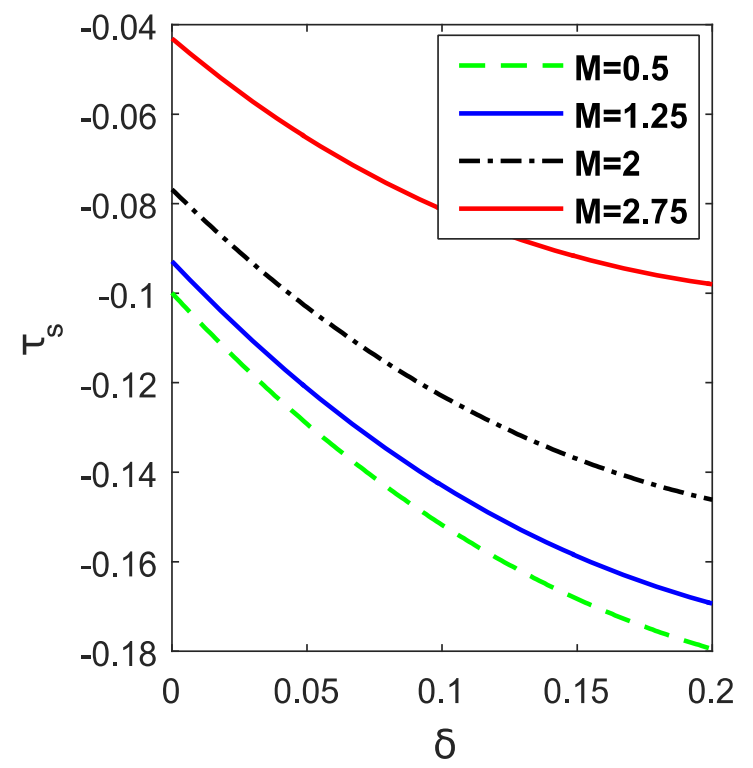

Fig.17. Variation of shear stress at the stenosis throat for different values of the magnetic field parameter $(M)$.

The expression for shearing stress at the maximum height of the stenosis i.e., shear stress at the stenosis throat located at $z=\frac{a}{b}+\frac{1}{n^{\frac{n}{n-1}}}$, can be defined as

$$
\tilde{\tau}_{s}=\left[\tilde{S}_{r z}\right]_{h=(1-\delta)} .
$$




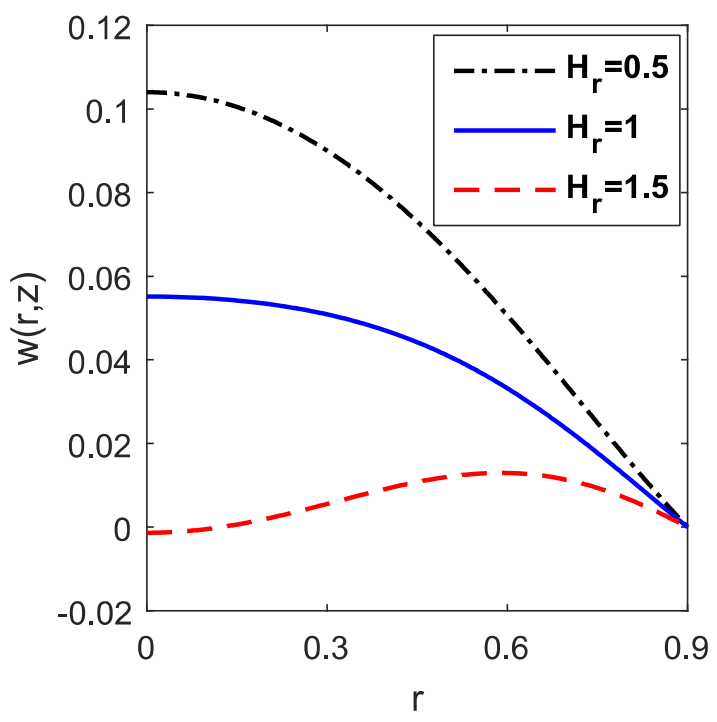

Fig.18. Radial distribution of velocity profile for different values of hematocrit parameter.

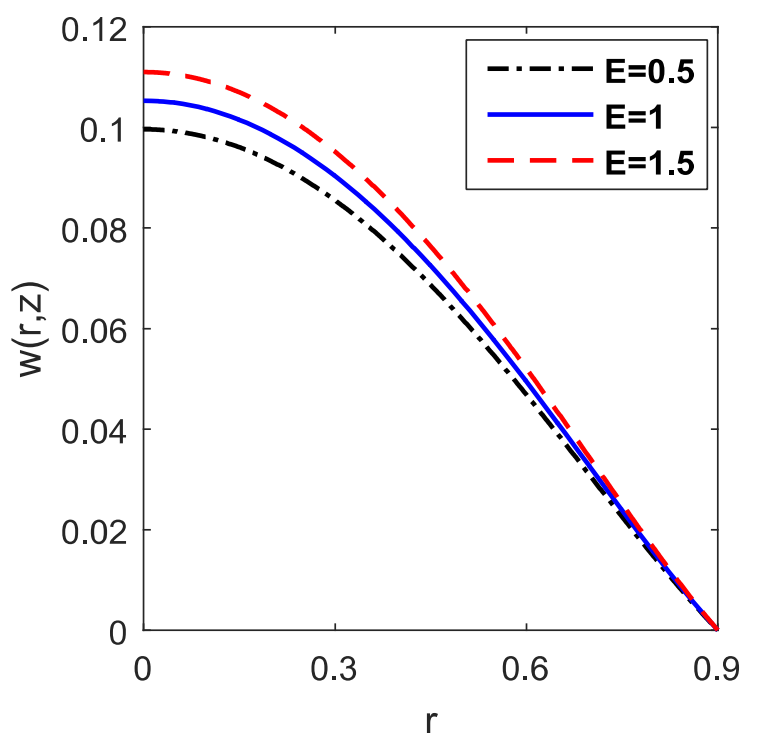

Fig.20. Variation of the velocity profile for different values of chemical reaction parameter.

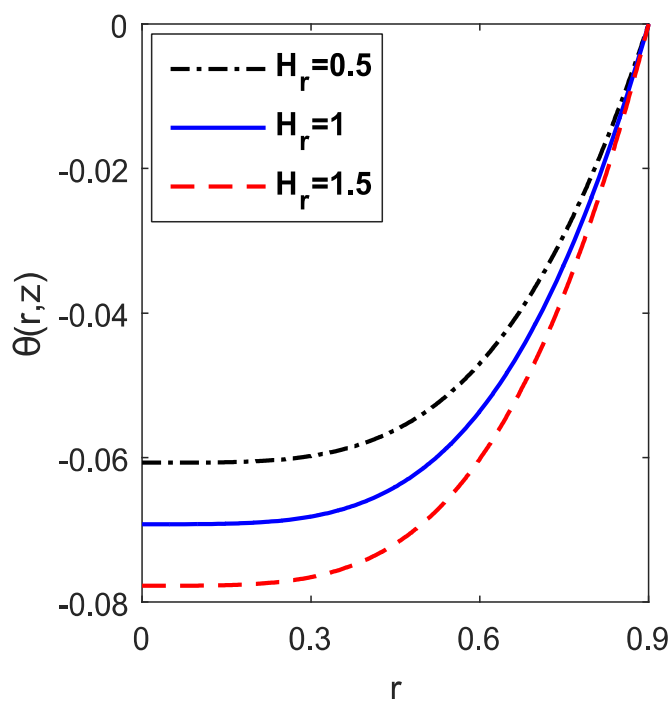

Fig.19. Radial distribution of temperature profile for different values of hematocrit parameter.

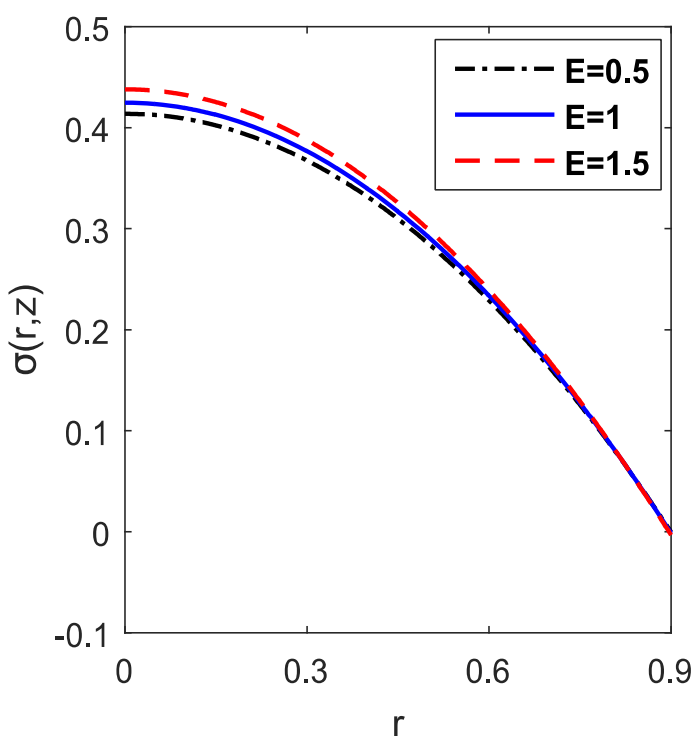

Fig.21. Variation of concentration profile for different values of chemical reaction parameter.

Figure 18 and Fig.19 are prepared to analyze the effects of the hematocrit parameter $\left(H_{r}\right)$ on velocity and temperature profiles of the blood flow in an inclined porous artery. Figure 18 shows that as the values of the hematocrit parameter increase, the velocity profile decreases, receptively. From Eq.(2.11) it is clear that the hematocrit ratio of the blood directly affects the viscosity of the blood flow. So as the number of red blood cells present in the blood volume increases, it will be relatively difficult for blood particles to move from one place to another place because of the higher viscosity and this results in the decreased value of the velocity profile of the blood flow. Figure 19 illustrates that as the values of the hematocrit parameter increase from 0.5 to 1.5 , the temperature profile of the blood flow decreases respectively.

Figures 20 and 21 focus on a variation of velocity and concentration profiles of the blood flow for different values of the chemical reaction parameter $(E)$. From Fig.20 it can be clearly observed that as the values of the chemical reaction parameter increase, the velocity profile also increases. Figure 21 indicates that as the values of the chemical reaction parameter increase, the concentration profile of the MHD blood 
flow in an inclined porous artery increases. Further, these figures reveal that for a fixed value of the chemical reaction parameter, both the velocity and concentration profiles decrease as we move gradually from the middle of the artery towards the stenosed arterial wall.

\section{Contour plots}
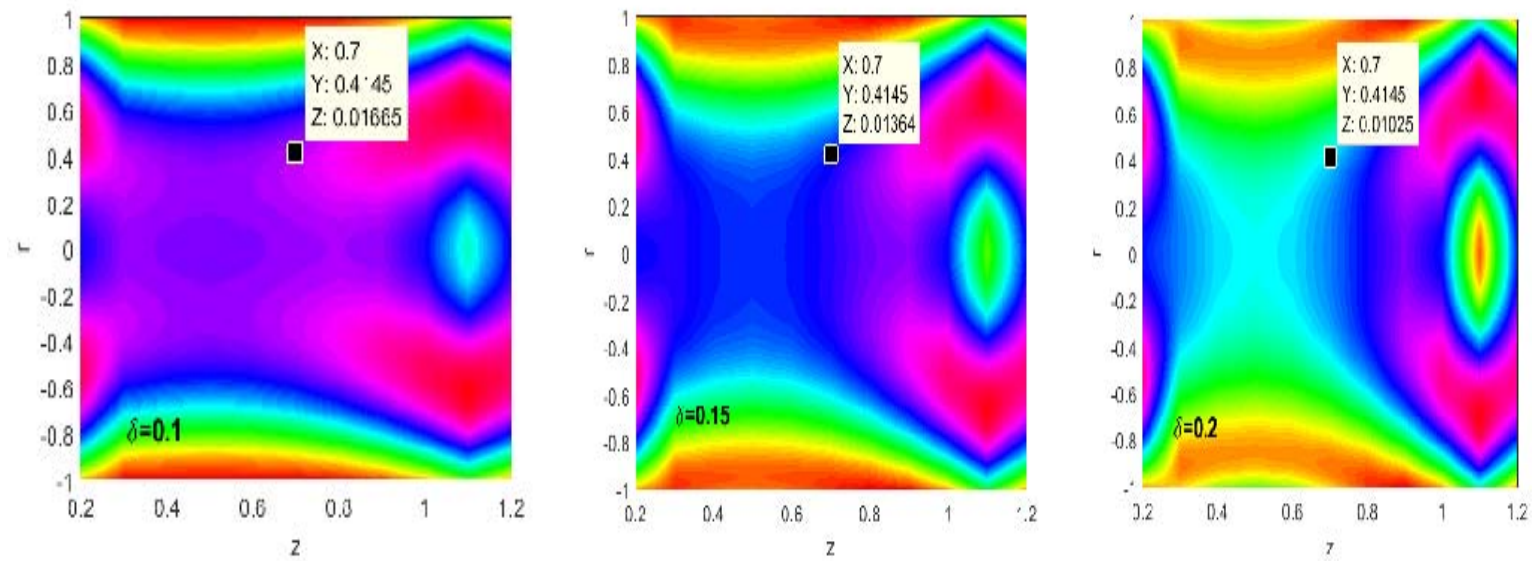

Fig.22. Contour plots of velocity for different values of the height of the stenosis $(\delta)$.
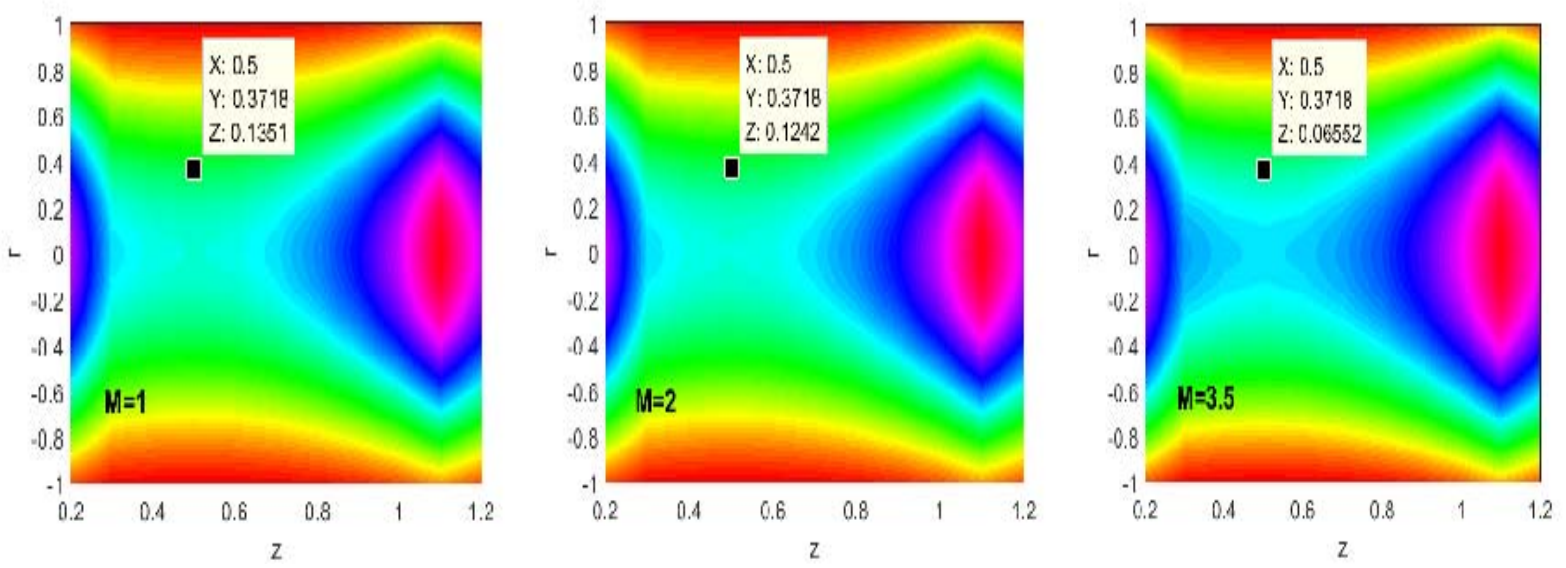

Fig.23. Contour plots of velocity for different values of the magnetic field parameter $(M)$.

Figures 22 and 23 are prepared (using the values of physical parameters as given in Tab.1) to display the contour plots for the variation of the velocity profile of the blood flow for different values of the height of the stenosis and the magnetic field parameter. In these figures, $X, Y, Z$ display the scale of the axial distance, radial distance and velocity at that point, respectively. Contours are plotted for the stenosis region range, $z=0.2$ to $z=1.2$, in which the maximum height of stenosis is located at $z=0.7$. So as the height of the stenosis $(\delta)$ increases, the size of the trapped bolus also increases and slowly it slows down the flow of blood at the maximum height of the stenosis. Figure 23 shows that as the effects of the magnetic field parameter increase, the trapped bolus also increases in size which gradually reduces the velocity of the blood flow. As it cleared that at point $(0.5,0.3718)$, the value of the velocity reduces from 0.1351 to 0.06552 as influence of the magnetic field increases from 1 to 3.5 .

\section{Conclusion}

In this investigation, effects of chemical reaction with heat and mass transfer have been analyzed on the blood flow of having variable viscosity through an inclined artery under the influence of applied 
magnetic field. Governing nonlinear differential equations have been solved by using homotopy perturbation method under the given boundary conditions. Effects of the physical parameters used in the problem such as the chemical reaction parameter $(E)$, porosity parameter $(Z)$, Schmidt number $(\mathrm{Sc})$, Soret number $(\mathrm{Sr})$, thermal Grashof number (Gr), solutal Grashof number $\left(G_{m}\right)$, hematocrit parameter $\left(H_{r}\right)$ on velocity, temperature and concentration profiles have been sketched graphically. Some effective findings of the article are summarized below

1. Blood velocity in the inclined artery increases as we increase the effects of porosity and chemical reaction parameters while it decreases when the values of the magnetic field and hematocrit parameters increase. This type of controlled behavior of the velocity profile under the effects of an applied magnetic field can help medical staff in their surgical procedures.

2. Wall shear stress in the stenosed inclined artery increases with a rise in the effects of the applied magnetic field. So due to this effect, high magnetic field strengths may cause the condition of plaque rupture that can affect the body by paralyzing the concerned portion of the body.

3. As the value of the inclination angle of the artery made from the vertical axis increases, the velocity of blood flow decreases while the temperature of the artery increases.

4. The height of the stenosis affects the velocity and temperature profiles of the blood flow in the sense that as the size of the stenotic region increases both the velocity and temperature profiles of the MHD blood flow decrease.

5. The concentration profile of the blood flow increases as the magnitude of the applied magnetic field parameter increases while it reduces with the increased value of the porosity parameter.

\section{Nomenclature}

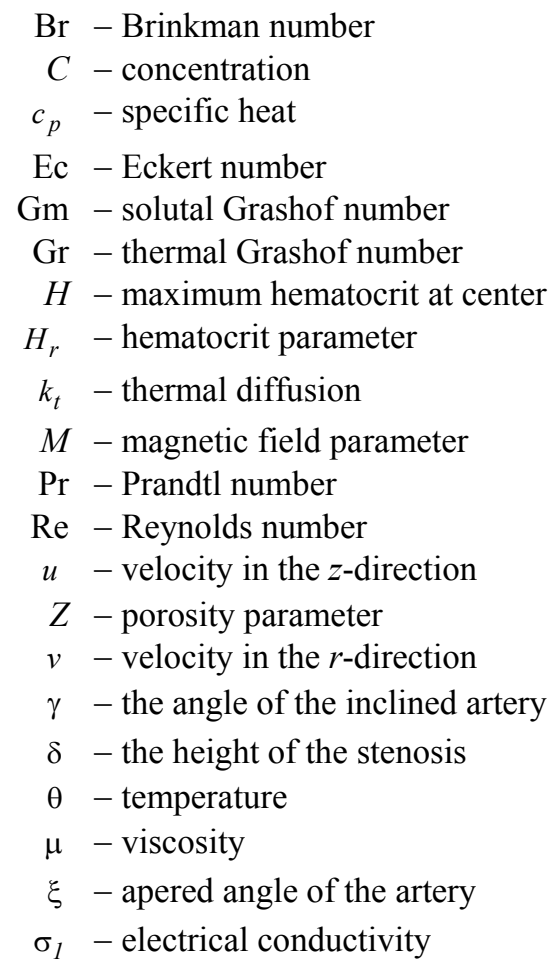

\section{Acknowledgment}

The authors are sincerely thankful to the Department of Science and Technology, Government of India (SR/FST/MSI-090/2013(C) ) for their financial support. 


\section{References}

[1] Ellahi R., Rahman S.U. and Nadeem S. (2014): Blood flow of Jeffrey fluid in a catherized tapered artery with the suspension of nanoparticles. - Physics Letters A, vol.378, No.40, pp.2973-2980.

[2] Rabby M.G., Razzak A. and Molla M.M. (2013): Pulsatile non-Newtonian blood flow through a model of arterial stenosis. - Procedia Engineering, vol.56, No.5, pp.225-231.

[3] Srivastava V.P. and Saxena M. (1997): Suspension model for blood flow through stenotic arteries with a cell-free plasma layer. - Mathematical Biosciences, vol.139, No.2, pp.79-102.

[4] Falk E., Prediman K.S. and Fuster V. (1995): Coronary plaque disruption. - Circulation, vol.92, No.3, pp.657-671.

[5] Fung Y.C. (2013): Biomechanics: mechanical properties of living tissues. - Springer Science and Business Media.

[6] Ellahi R., Rahman S.U., Gulzar M.M., Nadeem S. and Vafai K. (2014): A mathematical study of non-Newtonian micropolar fluid in arterial blood flow through composite stenosis. - Applied Mathematics and Information Sciences, vol.8, No.4, pp.1567-1573.

[7] Pralhad R.N. and Schultz D.H. (2004). Modeling of arterial stenosis and its applications to blood diseases. Mathematical Biosciences, vol.190, No.2, pp.203-220.

[8] Baldwin A.L. and Wilson L.M. (1994): Stationary red blood cells induce a negative charge on mucosal capillary endothelium. - American Journal of Physiology-Gastrointestinal and Liver Physiology, vol.266, No.4, pp.G685-G694.

[9] Haik Y., Pai V. and Chen C.J. (1999): Development of magnetic device for cell separation. - Journal of Magnetism and Magnetic Materials, vol.194, No.1, pp.254-261.

[10] Tzirtzilakis E.E. (2005): A mathematical model for blood flow in magnetic field. - Physics of Fluids (1994present), vol.17, No.7, 077103-077118.

[11] Srivastava N. (2014): Analysis of flow characteristics of the blood flowing through an inclined tapered porous artery with mild stenosis under the influence of an inclined magnetic field. - Journal of Biophysics, 2014:9 pages.

[12] Akbarzadeh P. (2015): Pulsatile magneto-hydrodynamic blood flows through porous blood vessels using a third grade non-Newtonian fluids model. - Computer Methods and Programs in Biomedicine, vol.126, pp.3-19.

[13] Eldesoky I.M.I. (2014): Unsteady MHD pulsatile blood flow through porous medium in stenotic channel with slip at permeable walls subjected to time dependent velocity (injection/suction). - Walailak Journal of Science and Technology, vol.11, No.11, pp.901-922.

[14] Khaled A.R.A. and Vafai K. (2003): The role of porous media in modeling flow and heat transfer in biological tissues. - International Journal of Heat and Mass Transfer, vol.46, No.26, pp.4989-5003.

[15] Shit G.C. and Majee S. (2015): Pulsatile flow of blood and heat transfer with variable viscosity under magnetic and vibration environment. - Journal of Magnetism and Magnetic Materials, No.388, pp.106-115.

[16] Layek G.C., Mukhopadhyay S. and Gorla R.S.R. (2009): Unsteady viscous flow with variable viscosity in a vascular tube with an overlapping constriction. - International Journal of Engineering Science, vol.47, No.5, pp.649-659.

[17] Sinha A. and Misra J.C. (2014): MHD flow of blood through a dually stenosed artery: Effects of viscosity variation, variable hematocrit and velocity-slip. - The Canadian Journal of Chemical Engineering, vol.92, No.1, pp.23-31.

[18] Makinde O.D. and Onyejekwe O.O. (2011): A numerical study of MHD generalized Couette flow and heat transfer with variable viscosity and electrical conductivity. - Journal of Magnetism and Magnetic Materials, vol.323, No.22, pp.2757-2763.

[19] Mekheimer K.S., Haroun M.H. and El Kot M.A. (2012): Influence of heat and chemical reactions on blood flow through an anisotropically tapered elastic arteries with overlapping stenosis. - Appl. Math, vol.6, No.2, pp.281-292.

[20] El-Sayed M., Akbar A.N.S. and Nadeem S. (2012): Influence of heat and chemical reactions on hyperbolic tangent fluid model for blood flow through a tapered artery with a stenosis. - Heat and Mass Transfer, vol.43, No.1, pp.69-94. 
[21] Misra J.C. and Adhikary S.D. (2016): MHD oscillatory channel flow, heat and mass transfer in a physiological fluid in presence of chemical reaction. - Alexandria Engineering Journal, vol.55, No.1, pp.287-297.

[22] Mohyud-Din S.T. and Noor M.A. (2009): Homotopy perturbation method for solving partial differential equations. - Zeitschrift für Naturforschung A, vol.64, No.(3-4), pp.157-170.

[23] Mekheimer K.S. and El Kot M.A. (2008): The micropolar fluid model for blood flow through a tapered artery with a stenosis. - Acta Mechanica Sinica, vol.24, No.6, pp.637-644.

[24] Shukla J.B., Parihar R.S. and Rao B.R.P. (1980): Effects of stenosis on non-newtonian flow of the blood in an artery. - Bulletin of Mathematical Biology, vol.42, No.3, pp.283-294.

[25] Sanyal D.C., Das K. and Debnath S. (2007): Effect of magnetic field on pulsatile blood flow through an inclined circular tube with periodic body acceleration. - Vol.11, pp.43-56.

[26] Ramesh K. (2016): Influence of heat and mass transfer on peristaltic flow of a couple stress fluid through porous medium in the presence of inclined magnetic field in an inclined asymmetric channel. Journal of Molecular Liquids, vol.219, pp.256-271.

[27] Bhatti M.M. and Abbas M.A. (2016): Simultaneous effects of slip and MHD on peristaltic blood flow of Jeffrey fluid model through a porous medium. - Alexandria Engineering Journal, vol.55, No.2, pp.1017-1023.

[28] Misra J.C. and Adhikary S.D. (2016): MHD oscillatory channel flow, heat and mass transfer in a physiological fluid in presence of chemical reaction. - Alexandria Engineering Journal, vol.55, No.1, pp.287-297.

[29] Nadeem S., Akbar N.S., Hayat T. and Hendi A.A. (2011): Influence of heat and mass transfer on Newtonian biomagnetic fluid of blood flow through tapered porous arteries with a stenosis. - Transport in Porous Media, vol.91, No.1, pp.81-100.

[30] Rao A., Sivaiah S. and Raju R.S. (2012): Chemical reaction effects on an unsteady MHD free convection fluid flow past a semi-infinite vertical plate embedded in a porous medium with heat absorption. - Journal of Applied Fluid Mechanics, vol.5, No.3, pp.63-70.

[31] Zaman A., Ali N., Bég O.A. and Sajid M. (2016): Heat and mass transfer to blood flowing through a tapered overlapping stenosed artery. - International Journal of Heat and Mass Transfer, vol.95, pp.1084-1095.

[32] Thompson B.L., Gilbert R.J., Mejia M., Shukla N., Haverstick D.M., Garner G.T. and Landers J.P. (2016): Hematocrit analysis through the use of an inexpensive centrifugal polyester-toner device with finger-to-chip blood loading capability. - Analytica Chimica Acta, vol.924, pp.1-8.

[33] Sharma S., Singh U. and Katiyar V.K. (2015): Magnetic field effect on flow parameters of blood along with magnetic particles in a cylindrical tube. - Journal of Magnetism and Magnetic Materials, vol.377, pp.395-401.

[34] Swartz R.H., Bhuta S.S., Farb R.I., Agid R., Willinsky R.A., Butany J., Wasserman B.A., Johnstone D.M., Silver F.L. and Mikulis D.J. (2009): Intracranial arterial wall imaging using high-resolution 3-tesla contrast-enhanced MRI. - Neurology, vol.72, No.7, pp.627-634.

[35] Misra J.C. and Shit G.C. (1988): Role of slip velocity in blood flow through stenosed arteries: a non-Newtonian model. - Journal of Mechanics in Medicine and Biology, vol.7, No.03, pp.337-353.

[36] Misra J.C. and Kar B.K. (1988): Momentum integral method for studying flow characteristics of blood through a stenosed vessel. - Biorheology, vol.26, No.1, pp.23-35.

[37] Kandasamy R., Periasamy K. and Prabhu K.K.S. (2005): Effects of chemical reaction, heat and mass transfer along a wedge with heat source and concentration in the presence of suction or injection. International Journal of - Heat and Mass Transfer, vol.48, No.7, pp.1388-1394. 